\title{
Investigation of Open Standards to Enable Interoperable Wide Area Monitoring for Transmission Systems
}

\author{
Mohammad Golshani \\ mohammad.golshani@brunel.ac.uk
}

\author{
Gareth A. Taylor \\ gareth.taylor@brunel.ac.uk
}

\author{
Ioana Pisica \\ ioana.pisica@brunel.ac.uk
}

\author{
Phillip Ashton \\ phillip.ashton@brunel.ac.uk
}

Brunel Institute of Power Systems, Brunel University, Uxbridge, Middlesex, UB8 3PH, UK

\begin{abstract}
Nowadays, power systems face more challenging network wide issues with regard to ensuring secure and reliable operation. Therefore, having a wide area monitoring system is a vital need in order to detect problems and react on them as quickly as possible. An important component when providing wide area monitoring and control for transmission networks is the SCADA system, which connects the substations to the control center by polling data from Remote Terminal Units (RTUs). However, due to data rate limitations, the monitoring is relatively static and therefore infrequent. By using Phasor Measurement Units (PMUs) data can be provided in higher rates and with higher accuracy. Since different manufacturers exist in the market, standardization is the key for advancement of the connectivity and interoperability within the system. In the past, utilities used to employ proprietary protocols, which were specified by the product vendors. Gradually, it was decided to move towards open standards to provide an interoperable environment and improve modelling capabilities. Apart from PMU standards, in a typical power system several communication protocols exist and are required for transferring data and each of them covering certain domains and specific groups of data. The objective of this paper is to investigate the adoption, development and performance of the most common open standards to enable interoperable wide area monitoring systems.
\end{abstract}

Index Terms-Communication Protocols, Open Standards, PMU, RTU, SCADA, WAMS

\section{INTRODUCTION}

$\mathrm{O}$ peration of power systems has become more complicated and therefore encounters more challenges, mainly due to deregulation of the power industry and an increase in the penetration of renewable energy sources. On the other hand, power electronics driven sensitive loads require a high level of reliability and power quality. In addition, difficulties also exist in upgrading the transmission system proportional to the growing generations and loads. These are some illustrations of why Wide Area Monitoring System (WAMS) must be deployed throughout the power system in order to observe, in real-time, the changing state of the electrical power network, take appropriate corrective measures and isolate faults quickly $[1,2]$.

Because of the extension of the power networks, suitable devices are required to enable synchronization between the remote instruments. Phasor Measurement Units (PMUs) are considered as the key component of WAMS [3]. PMUs measure power system parameters, including voltage and current phasors, with high accuracy and transmit the highly sampled values online to a central location called a Phasor Data Concentrator (PDC). The feature that makes PMUs more suitable for advanced monitoring and control of wide areas is the universal time stamp of measurements. This enables simultaneous measurement of system parameters from different locations in the power system, making the comparison of measured parameters simple, as depicted in Fig. 1. In fact, by using signals from Global Positioning System (GPS), the measurements can be precisely synchronized [2].

However, apart from the PMUs and PDCs, a high-speed and intelligent communication infrastructure is required for sending the time-critical data, alongside high performance software for viewing and analyzing of real-time and stored data [1]. Even though PMUs found their use mainly in transmission systems, the interest in highly accurate measurements has driven to their deployment at lower voltage levels. Current investigations are taking place in order to exploit PMUs benefits in distribution networks. PMUs have a significant role to play in the successful transition of today's huge amount of power delivery into a "Smart Transmission Grid" since the number of PMUs is growing in Europe and around the world [2, 4].

The high number of manufacturers active on the power system/WAMS market, each implementing proprietary protocols and applications could lead to systems that cannot be interconnected. Therefore, standardization is key for the advancement of the connectivity and interoperability within the system.

\section{SYNCHROPHASORS STANDARDS}

The first standard for synchrophasors, IEEE 1344, was introduced in 1995 and defined basic concepts for the measurement and method for data handling. However, technology is constantly evolving and standards should be updated in order to accommodate new requirements. Thus, the new standard IEEE C37.118 was published in 2005, which significantly improved the previous standard, while still maintained basic compatibility [5]. 


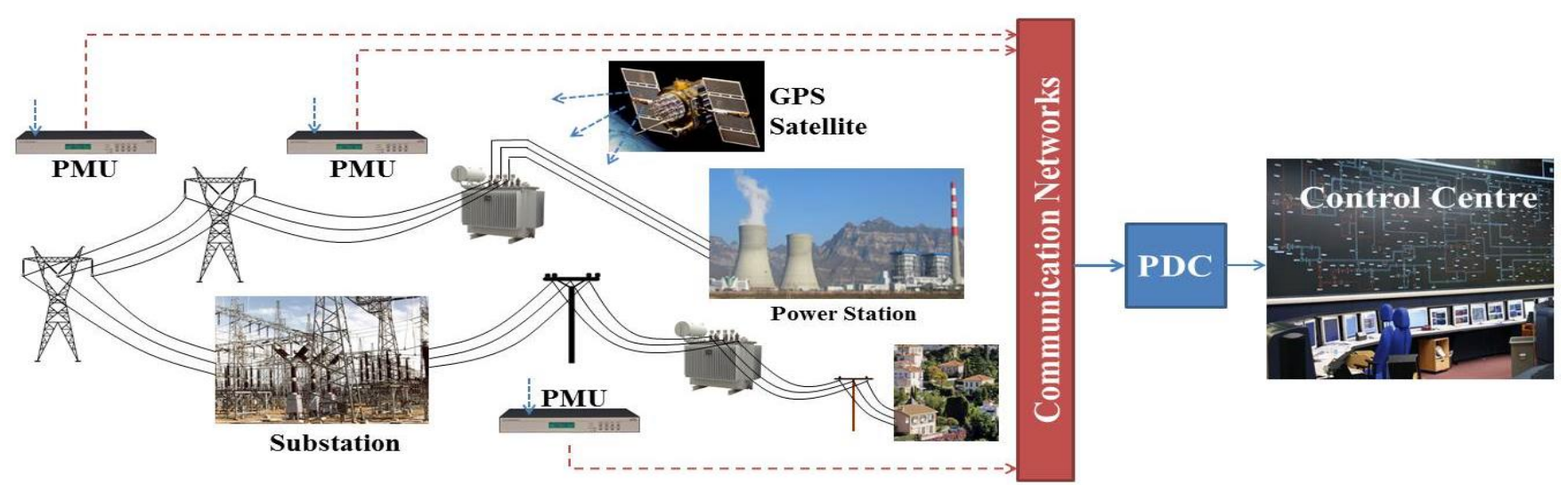

Fig. 1. Using PMUs in Wide Area Monitoring System [4]

The IEEE C37.118-2005 open standard specified a set of fundamental characteristics: Time Reference (UTC), Rate of Measurement, Phase Reference (cosine), Accuracy Metrics (Total Vector Error), and Communication Model (format of messages). By defining these specifications, the real-time and off-line processing of synchrophasor data from different measuring systems can be performed more easily [6]. Although the publication of IEEE C37.118-2005 was an important step in standardizing phasor measurements, the standard does not cover all aspects. For example, it does not specify PMU performance requirements under dynamic conditions, which could lead to PMUs using the same standard to show different results under transient situations. Moreover, it does not address frequency measurement requirements, and does not specify communication protocol, as it only defines the data format with basic methods for data transfer [5].

Due to the above issues, further work was done to revise the standard in 2008. In addition, IEC 61850 was proposed to be employed as a communication standard for transferring measured synchrophasors [7]. However, there were some problems in merging C37.118 into IEC 61850. As a solution to these issues, it was proposed to split the C37.118 standard into two parts. The two new revised standards were completed and published in December 2011. The first part, C37.118.1-2011, includes the synchrophasor measurement definitions and requirements, and the second part, C37.118.22011 includes a new standard for synchrophasor data transfer, which is designed to be compatible with the IEC 61850. Both standards have maintained features from the previous version, but with updates and additional provisions [8,9].

The IEC 61850 standard and other communication protocols have an essential role in enabling the interoperability of devices in WAMS. This paper will provide an investigation of these standards, with their benefits and drawbacks.

\section{ModBUS}

The Modbus transmission protocol was developed by Gould Modicon (now Schneider) for process control systems. Basically, Modbus is a simple, inexpensive, robust, and easy to use serial communications protocol that has become a de facto standard communication protocol in the industry since $1979[10,11]$.

In fact, Modbus is an application layer protocol positioned at level 7 of the Open Systems Interconnection (OSI) model that provides client/server communication for one server and up to 247 clients, which are connected to the different networks [12]. Transactions are either a query/response type where only a single client is addressed, or a broadcast/no response type where all clients are addressed [10]. In either case only the server initiates messages and in other words report by exception is not supported except for Modbus over Ethernet TCP/IP [12]. Therefore, the server must routinely poll each client to identify changes in the data. Accordingly, this occupies bandwidth and takes much time that is more significant where bandwidth is limited and expensive, such as over a low-bit-rate radio link.

Modbus is currently implemented using the following different transmission protocols [12]:

- TCP/IP over Ethernet

- Asynchronous serial transmission over variety of media (wire RS-232, 422 or 485, fiber, radio, etc.)

- Modbus Plus, a high speed token passing network (which is currently proprietary to Modicon)

For Asynchronous Modbus and Modbus Plus the application Data Unit (ADU) is directly mapped to the physical layer, while in Modbus Ethernet TCP/IP it is first passed through the transport and the network layers. Fig. 2 shows this concept [7]:

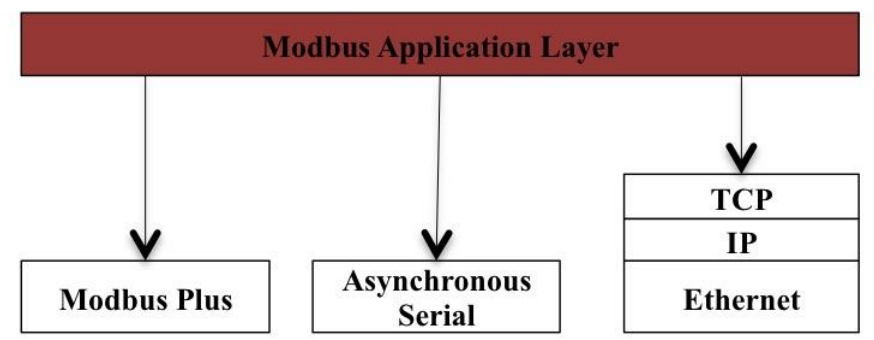

Fig. 2. Modbus communication stack

By using gateways, these three implementation types can exist in a communication network at the same time and the 
Modbus protocol enables all types of network architecture to communicate with each other in a simple manner [7].

Some characteristics of the Modbus protocol are fixed, such as frame format, frame sequence, dealing with communications errors and exception conditions, and the functions performed. On the other hand, other characteristics can be determined by the user. These are transmission medium, transmission characteristics and transmission modes (RTU or ASCII) [10].

A transaction comprises of a single request from the host to a particular secondary device and afterwards a single response from that device back to the host. Both of these messages are formatted as Modbus message frames. The Modbus protocol defines a simple protocol data unit (PDU) independent of the underlying communication layers for transmission of messages between server and clients. Fig. 3 shows general Modbus frame (bytes demonstrated here are in Hex format and not in ASCII) [10]:

\begin{tabular}{|c|c|c|c|}
\hline \multicolumn{4}{|c|}{ Application Data Unit (ADU) } \\
\hline \multicolumn{3}{|c|}{ Protocol Data Unit (PDU) } & \\
\hline 1 Byte & 1Byte & Variable & 2 Bytes \\
\hline $\begin{array}{c}\text { Address } \\
\text { Field }\end{array}$ & $\begin{array}{c}\text { Function } \\
\text { Field }\end{array}$ & Data Field & $\begin{array}{c}\text { Error-Check } \\
\text { Field }\end{array}$ \\
\hline
\end{tabular}

Fig. 3.Modbus frame format

As indicated, such message frames includes a series of bytes classified into four fields and they will be described in the following paragraphs:

A. Address Field

The first field of Modbus frame is the address field, which is composed of a single byte of information. In the request frame, this byte identifies the controller to which the request is being directed. The response frame begins with the address of the responding device. Theoretically, each client may have an address field between 1 and 247. However, practical limitations will limit the maximum number of clients [10].

\section{B. Function Field}

The second field in each frame is the function field, which is also composed of a single byte of information. When a request message is sent from a host to a target, the function code field tells the target device what kind of action to perform. If the target device is able to perform the requested function, the response frame will have the same function field as the original request. Otherwise, the function field of the response frame will be echoed with its most-significant bit set to one. Thus, signaling an exception response including an appropriate exception code that the host application can use to determine the next action to be taken. Valid function codes are in the range of 1 to 255 decimal, where the range 128-255 is reserved and applied for exception responses (function code " 0 " is not valid) [12].

\section{Data Field}

The third field in a message frame is the data field. The length of this byte is variable according to the function that is applied in the function field of the frame. In a host request, this field contains additional information that target devices use to take the action defined by the function code and in a target device response this field comprises of any data requested by the host. The data field may have zero length where server does not require any additional information and the function code alone specifies the action [12].

\section{Error-Check Field}

The last field of a message frame is the two bytes errorcheck field. The numeric value of this field is calculated by performing a cyclic redundancy check (CRC-16) on the message frame. During receipt of a message, the receiving device calculates a CRC and compares the calculated value to that which was calculated by the transmitting device. If the two values differ, an error will result.

In order to provide reliable communication, the messages reception must be synchronized with its transmission. In other words, the start of the new message frame must be recognizable for the receiving device. Under the Modbus RTU protocol, frame synchronization is established by limiting the elapsed time between receipts of characters. If three character times (approximately three milliseconds) elapse without a new character or completion of a frame, then the pending message will be discarded, and the next byte received will be treated as the address field of a new message frame [13].

\section{IEC 60870}

In 1988, the International Electrotechnical commission (IEC) started publishing a standard entitled 'IEC 870 telecontrol equipment and system'. The standard was developed and published progressively and was later renamed IEC 60870 , by adding the prefix 60 . There are six main parts in the standard of which part five is for transmission protocols. IEC 60870-5 was developed in a hierarchical manner in five core sections alongside four companion standards in order to define an open standard for SCADA communications and wide area processes. The IEC 60870 protocol is mainly used in the electrical industries of European countries, and has data objects that are specifically provided for such applications. However, it is not limited to electrical industries and has data objects that can be applied for general SCADA applications in any industry [14].

Primarily, the three-layer Enhanced Performance Architecture (EPA) was adopted as the basis for data transmission in the IEC 60870 standard. The EPA is the simplified three-layer sub-set of the OSI seven-layer model and consists of application, data link and physical layers. One layer is normally added to the top of the EPA model, which is defined as the "user process" layer. This extra layer represents the various functions or processes that must be specified to provide telecontrol system operations. Generally, the IEC 60870-5 document determines four frame formats that are used for telecontrol applications. These four frame formats are FT1.1, FT1.2, FT2 and FT3 [10, 15].

The companion standard IEC 60870-5-101 which is called 'Companion Standard for Basic Telecontrol Tasks' uses the FT1.2 frame format. In fact, when we discussed about IEC 60870 in the field of SCADA system, the IEC 60870-5-101 
part of the protocol has the key role. It provides the application level data objects that are required for SCADA operations.

IEC 60870-5 set of standards was initially published on the basis of IEC 60870-5-101 profile shown in Fig. 4. It covered only transmission over relatively low bandwidth bit-serial communication circuits. But after increasing of network communication applications, the fourth companion standard IEC 60870-5-104, was introduced in order to define the transport of IEC 60870-5 applications messages over networks using the TCP/IP protocol. As Fig. 5 shows, the IEC60870-5-104, which is titled 'Network Access using Standard Transport Profiles' provides a very different physical and data transport procedure compared to IEC 60870-5-101. In this protocol the lower levels of the protocol have been completely replaced by the TCP and IP transport and network protocols, respectively. However, it retained most of the higher application level functions and data objects as it was. TCP and IP protocols are applied for transport of the application service data units (ASDUs) over local area and wide area networks [10].

\begin{tabular}{|c|c|c|} 
Layer & Source & Description \\
\hline User Process & IEC 60870-5-5 & Application functions \\
\hline \multirow{2}{*}{ Application } & IEC 60870-5-4 & $\begin{array}{c}\text { Application information } \\
\text { elements }\end{array}$ \\
\cline { 2 - 3 } & IEC 60870-5-3 & ASDUs \\
\hline \multirow{2}{*}{ Link } & IEC 60870-5-2 & Transmission procedures \\
\cline { 2 - 3 } & IEC 60870-5-1 & Frame formats \\
\hline \multirow{2}{*}{ Physical } & ITU-T & Interface specification \\
\hline
\end{tabular}

Fig. 4. IEC 60870-5-101 communication stack [10]

\begin{tabular}{|c|c|c|}
\hline Layer & \multicolumn{1}{|c|}{ Source } & Description \\
User Process & IEC 60870-5-101 & Application functions \\
\cline { 1 - 1 } Application & IEC 60870-5-101 & $\begin{array}{c}\text { Application information } \\
\text { elements and ASDUs }\end{array}$ \\
\cline { 1 - 1 } Transport & \multicolumn{2}{|}{} \\
\cline { 1 - 1 } Network & \multicolumn{2}{|c}{ TCP/IP Protocol Suite } \\
\cline { 1 - 1 } Link & & \\
\cline { 1 - 1 } Physical & \\
\hline
\end{tabular}

Fig. 5. IEC 60870-5-104communication stack [10]

IEC 60870-5-102 and IEC 60870-5-103 companion standards provide data types and functions to support electrical protection systems. In this paper we have focused on T101 and T104 companion standards.

Whereas T101 provides full definition of the protocol stack right down to the physical level, this is not provided under T104 as existing and varied physical and link layer operations are employed.

IEC 60870-5-101 supports point-to-point and multidrop communication links carrying bit-serial low-bandwidth data communications. It also provides the choice of using balanced or unbalanced communication at the link level. Under unbalanced communication, only the master can initiate communications by transmitting primary frames. As the slaves are not able to initiate a transaction, the collision avoidance process is not required. Under T101 profile the balanced communication can be used only for the point-topoint links. This means that T101 profile cannot support unsolicited messages from slaves, in order to send data directly to the master, for the multidrop topologies. Therefore, it must adopt a cyclic polling procedure to inquire about the secondary stations [10].

\section{Message Structure}

The IEC 60870-5-101 message structure is formed by the data link layer and includes link address, control information, user data and so on. Each frame cannot carry more than one ASDU. Fig. 6 shows the data link layer frame and the structure of ASDU carried by it [10].

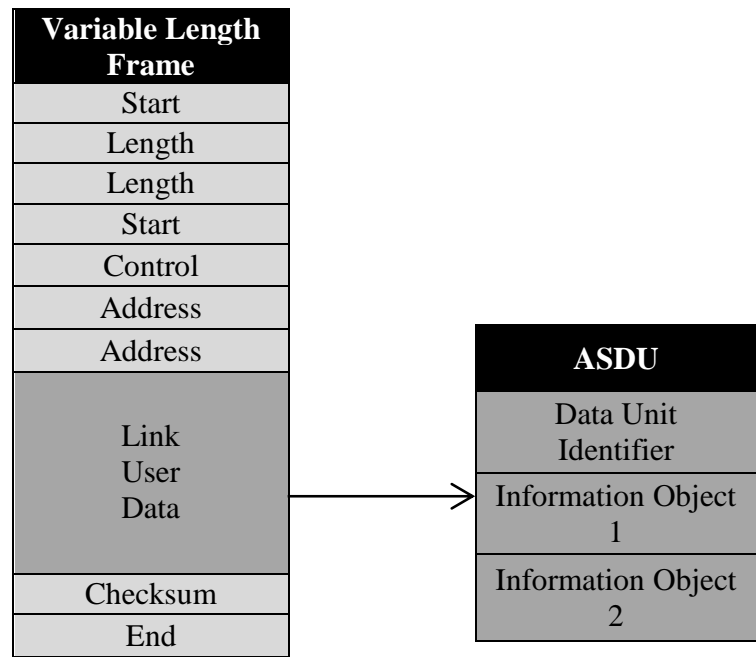

Fig. 6. Data link frame format

It should be mentioned that for the network version, T104 profile, the ASDU is carried by the TCP/IP protocols instead of the T101 link layer. Therefore, the link frame of Fig. 6 does not cover this part. This frame format will be briefly described in the following paragraphs.

The length section repeated twice, and the two values must be equal so that the whole frame can be considered as a valid one. The maximum frame length is 261 octets. However, a lower maximum frame length can be specified.

The control field of the data frame has a key role in operation of the transmission procedure. It depends on the modes of the transmission, balanced or unbalanced. In addition, the interpretation of the control field is dependent on whether the communication is a primary or secondary message [10].

Under T101 profile, addressing is both at the link and application level. The link address field may be 1 or 2 octets for unbalanced and 0,1 or 2 octets for balanced communication. Because balanced communication is only applied for point-to-point architectures the link address is not necessary and can be used just for security purposes. For addressing all stations, the link address FF or FFFF is used as a broadcast message [14].The structure of ASDU is divided to two main sections, the data unit identifier and the data itself (one or more information objects). Finally, for the security provisions, T101 uses an eight-bit checksum. 


\section{Distributed Network PROTOCOL VERSION 3 (DNP3)}

During the same period that IEC 60870-5 was gradually published, the DNP3 protocol was developed and introduced in North America. In fact, they originated from a common point provided by the early IEC 870 document. For instance, DNP3 uses FT3 format, which is one of the four frame formats defined by the IEC 60870 [10]. Nevertheless, they differ in many aspects of physical, data link and application functions. Initially, Harris Control Division created DNP3 as a proprietary protocol for electrical industry applications in the early 1990s. However, in November 1993 the protocol ownership was transferred to the DNP3 User Group in order to use it as an open standard in industry [7].

Both DNP3 and IEC 60870-5 were developed fundamentally for SCADA applications. These entail acquisition of information and sending of control commands between master stations, RTUs and other IEDs. They are designed in a way to transmit relatively small packets of data in a deterministic sequence and reliable manner. Hence they are distinct from more general purpose communication protocols, such as FTP. Whereas they can send quite large files, they are not suitable for SCADA applications [7].

DNP3 supports multi-slave, peer-to-peer and multiple master communications. It uses only balanced communications so it supports report by exception as well as polled operational mode. Report by exception capability enables the outstation devices to send unsolicited messages to the master station. This provides efficient use of the communication system capacity and greater flexibility. It should be noticed that although the outstation devices can initiate the communication in DNP3, only the master station could initiate a request for data or send commands [10].

DNP3 same as IEC 60870-5 is based on EPA model. However, it adds some kind of transport functions, which are represented as a layer named "pseudo-transport". This layer is located below the application layer and provides the transmission of larger data blocks than data link layer. Fig. 7 shows the modified EPA model for DNP3 implementation alongside the message build up structure. Each layer offers some services to the layer above it and adds information header to the messages blocks that are transferred to the lower layers. In the following paragraphs the overall messaging sequence of DNP3 protocol will be briefly described.

At the highest layer of the stack, application layer breaks down the data into smaller sized blocks, which are called application service data units (ASDUs). Then this layer adds the application header, application protocol control information (APCI), to each chunk and builds the application protocol data unit (APDU). The maximum size of each APDU is 2048 bytes, but the number of APDU required to present an ASDU is not limited. The APDU passed to the pseudo-transport layer are called transport service data unit (TSDU). TSDUs are broken down to the smaller blocks, which are called transport protocol data units (TPDUs). Each TPDU is made up of one byte of header and a maximum of 249 bytes for data. The data link layer receives the overall 250 bytes of TPDUs from the pseudo-transport layer [10].

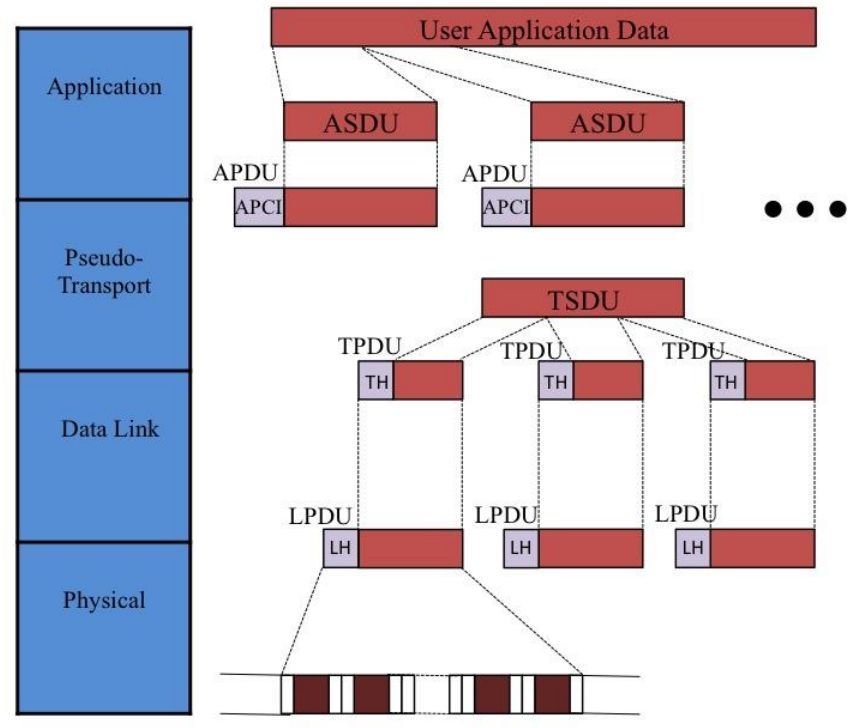

Fig. 7. DNP3 communication stack and data unit structures

According to the FT3 frame format, it will add a 10 bytes header to each including 2 bytes cyclic redundancy code (CRC) for providing error detection. These 2 bytes CRC will be repeated for each block of data in LPDUs. The maximum number of data blocks in LPDU is 16. Each block consists of 16 bytes of data (Except last block that may have less than 16 bytes according to the data size) and 2 bytes of CRC. The LPDU maximum size is 292 bytes, of which 250 bytes are data. Finally, the physical layer sends data as bit stream over the determined physical media [7].

Due to the need for operating over larger geographical areas, it has been proposed to use internet protocol suite and Ethernet for DNP3. In this case the transport, network and data link layer related to the TCP/UDP, IP and Ethernet LAN will be added at the bottom of the pseudo-transport and data link layer of DNP3 [7].

\section{IEC 61850}

IEC 61850 is a communication standard released by the Technical Committee (TC) 57 of IEC. It was originally introduced for the design of Substation Automation (SA). It defines communication between IEDs in substations and related system requirements. As a consequence of employing advanced and fast devices, the efficient and high-speed communication infrastructure has become an important issue in the substations [16]. The IEC 61850 standard has enabled IEDs and devices in a substation to be integrated on a highspeed peer-to-peer communication network as well as client/server communication [17].

IEC 61850 applies object oriented (OO) data and service models to support all substation functions. This provides more flexibility to the developer and users, simplifying engineering tasks [17]. In the IEC 61850 standard, protection and control functions are broken down into smaller units called Logical Nodes (LNs). In fact, these virtual units are the objects specified in the OO approach of the standard. Different functions in substations can be represented with 
these LNs. This is one of the most important advantages of the standard over legacy protocols [18].

The dominant architectural construct that IEC 61850 adopts is the abstracting technique. This feature provides the definition of objects that are independent of any underlying communication protocols. In other words, "Abstract" means that the standard only determines what the services are intended to provide, rather than how they are built. Therefore, the system will be compatible with the future developments in the communication technology. Afterwards, the abstract model is mapped to a specific protocol stack that can meet the data and services requirements. Fig. 8 shows the five types of communication services provided by IEC 61850. Sampled Value (SV) and Generic Object Oriented Substation Event (GOOSE) are mapped directly to the data link layer. Therefore, they eliminate processing of any middle layers and increase the performance. Generic Substation State Event (GSSE) is mapped to its own protocol profile. Client/Server communication uses the mapping of the application model to Manufacturing Messaging Specification (MMS) [19].

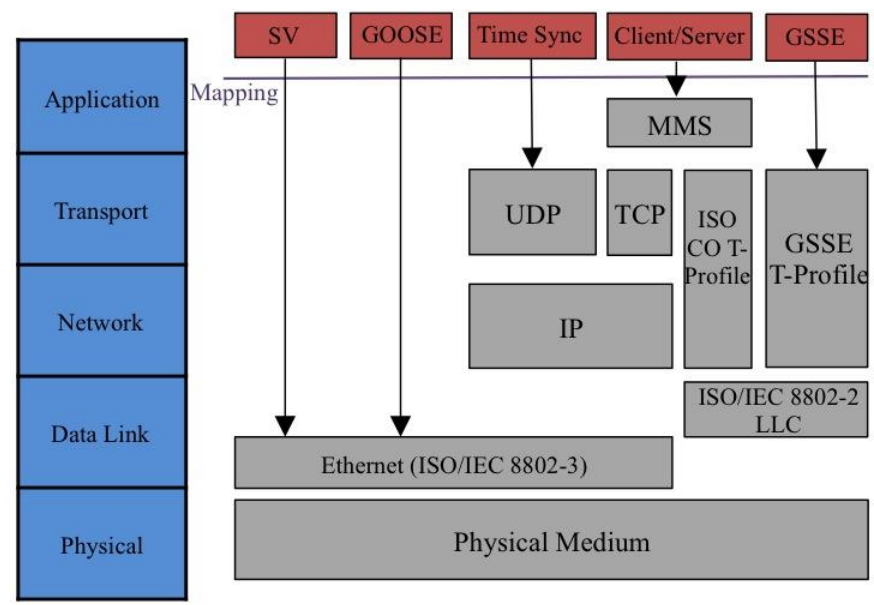

Fig. 8. Communication services defined in IEC 61850

IEC 61850 was originally designed in a way to operate over Ethernet and modern networks. While legacy protocols that were described in the previous sections, were basically designed for traditional serial link technologies. Hence due to the low bandwidth they adopted a procedure to reduce the transmitting data bytes. Despite this they were equipped with an Ethernet layer, they could not benefit efficiently from the wider bandwidth, as they are using the same procedure for data structure [19].

Although the scope of IEC 61850 was initially limited to the inside of substations, it is believed that the capabilities of IEC 61850 can be used to improve wide area communication applications. The Integration of IEC 61850 for PMU communications is one of the proposed applications [20].

\section{CONCLUSION}

Wide area monitoring is one of the vital requirements in developing of smart grid concept in power systems. In this regard, phasor measurement units can improve the monitoring by providing precise measurement in near realtime. A high-speed and proper communication infrastructure is the key to transfer phasor measurement data to the remote control centers and make time-critical wide area measurement applications feasible. As there are many manufacturers in the market, open standards should be employed to provide interoperability between equipment from different manufacturers. In this paper, some common open standard protocols were investigated. Among them IEC 61850 has some features that make it dominant, especially in timecritical applications.

\section{REFERENCES}

[1] Ballance, J.W., Bhargava, B., Rodriguez, G.D., "Monitoring Power System Dynamics using Phasor Measurement Technology for Power System Dynamic Security Assessmet," in Proc. IEEE Bologna Power Tech Conference, Vol. 3, June 2003, pp. 1-7.

[2] Gurbiel, M., Komarnicki, P., Styczynski, Z.A., Kereit, M. Blumschein, J., Buchholz, B.M., "Usage of Phasor Measurement Units for Industrial Applications," in Proc. Power and Energy Society General Meeting, July 2011, pp. 1-5.

[3] Lixia, M., Muscas, C., Sulis, S., "On the accuracy specifications of Phasor Measurement Units," in Proc. Instrumentation and Measurement Technology Conference, May 2010, pp. 1435-1440.

[4] L. Vanfretti and J.H. Chow, "Synchrophasor Data Applications for Wide-Area Systems,"In Proceedings of the Power Systems Computation Conference 2011, Stockholm, Sweden.

[5] Martin, K.E., "Synchrophasors Standards Development-IEEE C37.118 \& IEC 61850," in Proc. 44th Hawaii International Conference on System Sciences (HICSS), Kauai, HI, Jan. 2011, pp. 1-8.

[6] Vandiver, B., Apostolov, A., Steinhauser, F., "Testing of Phasor Measurement Units", in Proc. 63 ${ }^{\text {rd }}$ Annual Conference for Protective Relay Engineers, March/April 2010, pp. 1-5.

[7] Mohagheghi, S., Stoupis, J., Wang, Z., "Communication Protocols and Networks for Power Systems-Current Status and Future Trends," in Proc. Power Systems Conference and Exposition, Mar. 2009, pp. 1-9.

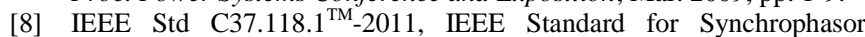
Measurements for Power Systems, Dec. 2011.

[9] IEEE Std C37.118.2 $2^{\mathrm{TM}}-2011$, IEEE Standard for Synchrophasor Data Transfer for Power Systems, Dec. 2011.

[10] G. Clarke, D. Reynders, Practical Modern SCADA Protocols. Burlington, MA: Elsevier, 2004.

[11] Using Modbus for Process Control and Automation, MOORE INDUSTRIES, pp. 1-7, [Online] available at http://www.isa.org.

[12] Modbus Application Protocol Specification V1.1b, Modbus-IDA, December 2006, [Online] available at http://www.modbus- IDA.org.

[13] Modbus Communication Module Functional Specification, OZTEK, January 2011,[Online] available athttp://www.oztekcorp.com.

[14] Peng Zhang, Advanced Industrial Control Technology. Burlington, MA: Elsevier, 2010.

[15] Medina, V.; Gomez, I.; Oviedo, D.; Dorronzoro, E.; Martin, S.; Benjumea, J.; Sanchez, G., "IEC-60870-5 application layer over TCP/IP for an open and flexible remote unit,"Industrial Electronics, IEEE International Symposium on, pp.420-425, 5-8 July 2009.

[16] IEC 61850-Communication Networks and Systems in Substations, Part 1- Introduction and Overview, IEC Std. 2003.

[17] Ozansoy, C.R.; Zayegh, A.; Kalam, A., "The Application-View Model of the International Standard IEC 61850,"Power Delivery, IEEE Transactions on, vol.24, no.3, pp.1132-1139, July 2009.

[18] Mohagheghi, S.; Tournier, J.-C.; Stoupis, J.; Guise, L.; Coste, T.; Andersen, C.A.; Dall, J., "Applications of IEC 61850 in distribution automation,"Power Systems Conference and Exposition (PSCE), IEEE/PES, pp.1-9, 20-23 March 2011.

[19] Mackiewicz, R.E., "Overview of IEC 61850 and Benefits,"Power Systems Conference and Exposition 2006, IEEE PES, pp.623-630, Oct. 29 2006-Nov. 12006.

[20] Pallares-Lopez, V.; Moreno-Munoz, A.; Gonzalez-Redondo, M.; RealCalvo, R.; Garcia, I.M.; de la Rosa, J.J.G., "Synchrophasor integration in IEC 61850 standard for Smart Grid and synchronism with PTP-base system,"Industrial Electronics and Applications (ICIEA), 2011 6th IEEE Conference on, pp.1507-1512, 21-23 June 2011. 